\title{
Noninvasive follicular thyroid neoplasm with papillary-like nuclear features: a single-institutional experience in Japan
}

\author{
Mitsuyoshi Hirokawa ${ }^{1)}$, Miyoko Higuchi ${ }^{2)}$, Ayana Suzuki ${ }^{2)}$, Toshitetsu Hayashi ${ }^{1)}$, Seiji Kuma ${ }^{1)}$ \\ and Akira Miyauchi ${ }^{3)}$ \\ 1) Department of Diagnostic Pathology and Cytology, Kuma Hospital, Kobe 650-0011, Japan \\ 2) Department of Laboratory Medicine, Kuma Hospital, Kobe 650-0011, Japan \\ 3) Department of Surgery, Kuma Hospital, Kobe 650-0011, Japan
}

\begin{abstract}
Noninvasive encapsulated follicular variant of papillary thyroid carcinoma (PTC) was reclassified as noninvasive follicular thyroid neoplasm with papillary-like nuclear features (NIFTP). To date, no studies on NIFTP have been reported in Japan. This study aimed to evaluate the clinical, cytological, and pathological findings of 54 cases of NIFTP from a single center in Japan, and compare them with those in the western countries. There were no significant differences in age, sex, or tumor size between patients with NIFTP and those with invasive encapsulated follicular variant -PTC. Ultrasound investigation showed a high suspicion lesion in $6.5 \%$ of NIFTP and $44.1 \%$ of invasive encapsulated follicular variant -PTC $(p<0.001)$. On fine needle aspiration cytology, $75.7 \%$ of NIFTP cases were reported as suspicious for malignancy or malignant. Nuclear grooves and irregular-shaped nuclei were observed in $94.6 \%$ of cases of NIFTP. Pathologically, $27.8 \%$ cases of NIFTP and $13.0 \%$ cases of invasive encapsulated follicular variant -PTC had been originally diagnosed as macrofollicular variants of PTC. There were no NIFTP cases with nodal metastasis. We concluded that NIFTP should be renounced noninvasive encapsulated follicular variant -PTC, and should be considered as a malignant tumor with exceeding indolent behavior, and lobectomy alone should be satisfactory for the diagnosis and treatment.
\end{abstract}

Key words: Thyroid, Noninvasive follicular thyroid neoplasm with papillary-like nuclear features, Macrofollicular variant, Aspiration cytology

RECENTLY, noninvasive encapsulated follicular variant (FV) of papillary thyroid carcinoma (PTC) was reclassified as noninvasive follicular thyroid neoplasm with papillary-like nuclear features (NIFTP) [1, $2]$. Because the vast majority of cases of NIFTP have indolent outcomes, adopting a new term indicating the nonmalignant nature of the neoplasm could avoid overtreatment and reduce burden of cancer diagnosis on patients [2]. The incidences of NIFTP range from $13.6 \%$ to $25 \%$ of PTC cases in the western countries [1], but have been reported to be as low as $0.8 \%$ in the Asian countries $[3,4]$. As revealed by fine needle aspiration cytology (FNAC), NIFTP is different from invasive FV-PTC, and most cases of NIFTP are classified as benign, atypia of undetermined significance or follicular lesion of undetermined significance (AUS/FLUS),

Submitted May 19, 2017; Accepted Aug 4, 2017 as EJ17-0214 Released online in J-STAGE as advance publication Sep. 12, 2017 Correspondence to: Mitsuyoshi Hirokawa, M.D., Ph.D., Department of Diagnostic Pathology and Cytology, Kuma Hospital, 8-2-35 Shimoyamate-dori, Chuo-Ku, Kobe, Hyogo 650-0011, Japan. E-mail: mhirokawa@kuma-h.or.jp

(C) The Japan Endocrine Society or follicular neoplasm or suspicious for a follicular neoplasm (FN/SFN) [5]. In contrast, Hahn et al. reported that the most common diagnosis of NIFTP was suspicious for malignancy (SFM), and there were no differences in the diagnosis of SFM and malignant between NIFTP and FV-PTC [6]. Thus, the incidences of these diseases and the impact of the reclassification on cytopathology outcomes are still controversial. In addition, no studies on NIFTP have been reported in Japan.

Herein, we report 54 cases of NIFTP studied at only one institute. This study aimed to evaluate clinical, cytological, and pathological findings of NIFTP in Japan, and compare them with those in the western countries. We also aimed to renounce the significance of the term NIFTP.

\section{Materials and Methods}

We reviewed 10,076 cases of resected and histologically diagnosed PTC at Kuma Hospital between January 2007 and June 2016. Among them, 284 cases (2.8\%) were diagnosed as FV-PTC, which included 107 
(1.1\%) cases of non-encapsulated FV-PTCs and 177 $(1.8 \%)$ cases of encapsulated FV-PTCs. Encapsulated FV-PTCs were divided into two groups-NIFTP (54 cases, $0.5 \%$ ) and invasive FV-PTC (123 case, 1.2\%). The criteria for diagnosis of NIFTP were as follows: 1) encapsulation or clear demarcation; 2) follicular growth pattern with less than $1 \%$ papillae; and 3) nuclear features of PTC. The nuclear features of PTC were grouped into three categories: 1) size and shape (nuclear enlargement/overlapping/crowding, elongation); 2) nuclear membrane irregularities (irregular contours, grooves, pseudoinclusions); and 3) chromatin characteristics (clearing with margination/glassy nuclei); the presence of two or three categories was required for the diagnosis [1]. Exclusion findings were as follows: 1) psammoma bodies; 2) more than 30\% solid, trabecular, or insular growth pattern; 3) vascular or capsular invasion; 4) tumor necrosis; and 5) high mitotic activity ( $\geq 3$ mitoses per 10 high-power fields). We compared cases of NIFTP and invasive encapsulated FV-PTC based on clinical and pathological findings. The presence of nodal metastasis was microscopically examined for regional lymph nodes removed at the time of thyroidectomy. Distant metastasis was evaluated during follow-up at 12 to 115 months (mean; 60 months) in cases of NIFTP, and at 13 to 122 months (mean; 68 months) in cases of invasive encapsulated FV-PTC. Immunohistochemical staining of Ki-67 (MIB-1; 1:200 dilution; Dako, Glostrup, Denmark) was performed using an automated Leica Bond-Max system and Bond Refine detection kit (Leica Microsystems, Wetzlar, Germany) according to the manufacturer's recommendations. The Ki-67 labeling index (LI) was estimated by counting at least 500 carcinoma cells.

Of the 54 NIFTP cases, 41 underwent pre-operative thyroid FNAC. FNAC was performed using a 22-gauge needle under ultrasound (US) guidance. Cytological slides were prepared by dispensing the aspirated materials from the needle onto glass slides and compressing them with a second slide; samples were then immediately fixed with Cytorop (Alfresa Pharma Co., Osaka, Japan), a cytological fixative. Samples were then stained using the Papanicolaou method. FNAC results were categorized into nondiagnostic or unsatisfactory (ND/UNS), benign, AUS/ FLUS, FN/SFN, SFM, or malignant, based on the criteria of the Bethesda System for Reporting Thyroid Cytopathology (BSRTC) [7]. The cytological findings of the specimens were reviewed.
Statistical analysis was carried out by Fisher's exact test or Student's $t$-test. Results with $p$ values less than 0.05 were considered statistically significant.

\section{Results}

Clinical and pathological findings of cases of NIFTP and invasive encapsulated FV-PTC are given in Table 1. Patients with NIFTP included 18 men and 36 women, with mean age of 60.8 years (range, 28-79 years). Compared to encapsulated invasive FV-PTC, NIFTP was more frequently noted in men; however, this

Table 1 Clinical and pathological findings of encapsulated follicular variant of papillary thyroid carcinoma

\begin{tabular}{|c|c|c|}
\hline & $\begin{array}{c}\text { Non-invasive } \\
\text { FV-PTC (NIFTP) } \\
(54)\end{array}$ & $\begin{array}{c}\text { Invasive } \\
\text { FV-PTC } \\
(123)\end{array}$ \\
\hline Age (yr)(mean) & $28-79(60.8)$ & $26-81(59.8)$ \\
\hline Gender (male/female) & $18 / 36$ & $25 / 98$ \\
\hline Size $(\mathrm{mm})($ mean $)$ & $4-50(17.5)$ & $5-58(17.4)$ \\
\hline Ultrasound interpretation & (46) & (118) \\
\hline High suspicion & $3(6.5 \%)$ & $52(44.1 \%)$ \\
\hline Intermediate suspicion & $14(30.4 \%)$ & $44(37.3 \%)$ \\
\hline Low suspicion & $24(52.2 \%)$ & $14(11.9 \%)$ \\
\hline Calcified nodule & $5(10.9 \%)$ & $8(6.8 \%)$ \\
\hline FNAC & (41) & (115) \\
\hline ND/UNS & $2(4.9 \%)$ & $3(2.6 \%)$ \\
\hline Benign & $2(4.9 \%)$ & $5(4.3 \%)$ \\
\hline AUS/FLUS & $5(12.2 \%)$ & $31(27.0 \%)$ \\
\hline FN/SFN & $1(2.4 \%)$ & $0(0 \%)$ \\
\hline SFM & $4(9.8 \%)$ & $20(17.4 \%)$ \\
\hline Malignant & $27(65.9 \%)$ & $56(48.7 \%)$ \\
\hline Macrofollicular variant & $15(27.8 \%)$ & $16(13.0 \%)$ \\
\hline Vascular invasion & $0(0 \%)$ & $2(1.6 \%)$ \\
\hline Ki-67 labeling index $>5 \%$ & $2 / 32(6.3 \%)$ & $2 / 62(3.2 \%)$ \\
\hline Nodal metastasis ${ }^{\#}$ & $0 / 22(0 \%)$ & $41 / 84(48.8 \%)$ \\
\hline Distant metastasis ${ }^{\#}$ & $0 / 22(0 \%)$ & $3 / 84(3.6 \%)$ \\
\hline
\end{tabular}

FV-PTC; follicular variant of papillary thyroid carcinoma, NIFTP; non-invasive follicular thyroid neoplasm with papillarylike nuclear features, FNAC; fine needle aspiration cytology, ND/UNS; nondiagnostic or unsatisfactory, AUS/FLUS; atypia of undetermined significance or follicular lesion of undetermined significance, FN/SFN; follicular neoplasm or suspicious for a follicular neoplasm, SFM; suspicious for malignancy $*<0.01, * *<0.001$, \# Multifocal PTC cases were excluded. 
difference was not statistically significant. The size of the tumors was measured by US. The largest dimension of NIFTP ranged from 4 to $50 \mathrm{~mm}$ (mean: 17.5 $\mathrm{mm}$ ) and was not significantly different from that of invasive encapsulated FV-PTC. US, before FNAC, revealed that three (6.5\%), 14 (30.4\%), and $24(52.2 \%)$ of the 46 NIFTP cases were categorized as high, intermediate, and low suspicion, respectively. The incidence of high suspicion cases of NIFTP was significantly lower than those of invasive encapsulated FV-PTC $(p<0.001)$, and the incidence of low suspicion cases of NIFTP was higher than that of cases of invasive encapsulated FV-PTC $(p<0.001)$. Five $(10.9 \%)$ cases of NIFTP could not be interpreted due to presence of marked calcified nodules.

According to the original cytology reports, of the 41 NIFTP samples assessed by FNAC, two (4.9\%), two (4.9\%), five (12.2\%), one (2.4\%), four $(9.8 \%)$, and 27 $(65.9 \%)$ were categorized as ND/UNS, benign, AUS/ FLUS, FN/SFN, SFM, and malignant, respectively. Of the 115 cases of encapsulated invasive FV-PTC, 56 $(48.7 \%)$ were reported as malignant. NIFTP was more likely to be diagnosed as a malignancy than as invasive encapsulated FV-PTC.

Pathologically, 15 (27.8\%) cases of NIFTP and 16 (13.0\%) cases of invasive encapsulated FV-PTC had been originally diagnosed as macrofollicular variants of PTC (Table 1, Fig. 1). The incidence of macrofollicular variants in cases of NIFTP was significantly higher than that in cases of invasive encapsulated FV-PTC ( $p$ $<0.01)$. There were no cases of NIFTP with vascular invasion. Ki-67 labeling indexes of more than $5 \%$ were observed in $6.3 \%$ of cases of NIFTP; this was not significantly different from that of invasive encapsulated FV-PTC. For evaluation of nodal and distant metastases, cases of multifocal PTC were excluded. All the 22 cases of NIFTP exhibited neither nodal metastasis at the time of the operation nor distant metastasis during follow-up. Invasive encapsulated FV-PTCs were associated with nodal metastasis in $48.8 \%$ of the cases. Three cases $(3.6 \%)$ of invasive encapsulated FV-PTC metastasized to the lung and/or bone.

Table 2 shows the cytological findings of 37 cases of NIFTP. In eight (21.6\%) cases of NIFTP, there were abundant colloids in the background (Fig. 2a). All cases had been diagnosed as macrofollicular variants of PTC on original histological examination. In 28 cases, the colloid materials were present on the preparations and appeared as lumps of various sizes that were inspis- sated and deeply stained. Large-, medium-, and smallsized lumps were from macrofollicular, normofollicular, and microfollicular clusters, respectively (Fig. 2). The former tended to be isolated from the cell clusters, and the latter two appeared within the cell clusters. Macrofollicular, normofollicular, and microfollicular patterns were observed in $16.2 \%, 24.3 \%$, and $83.8 \%$ of
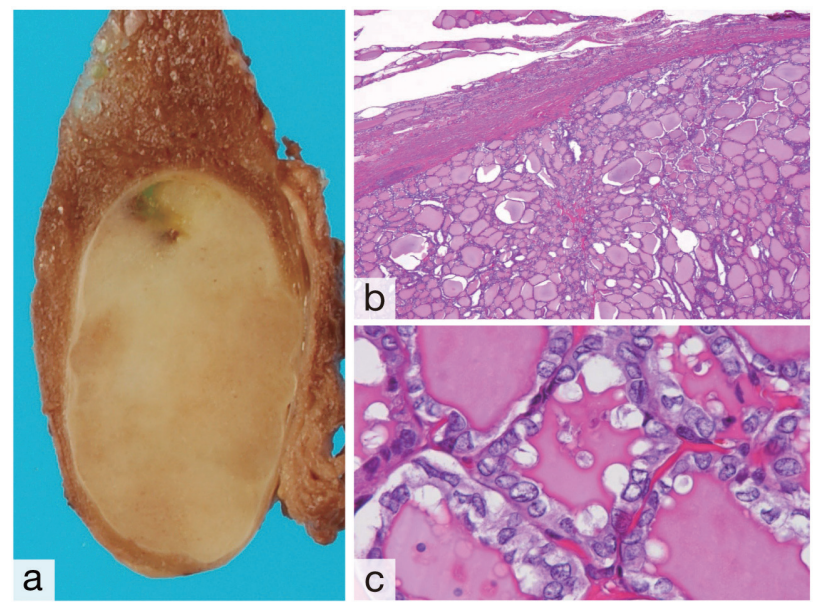

Fig. 1 Gross and microscopic findings of NIFTP that was originally diagnosed as a macrofollicular variant of papillary thyroid carcinoma. (a: Gross; b: H\&E stain, 40×; c: H\&E stain, 400×).

Table 2 Cytological findings of 37 NIFTP cases

\begin{tabular}{|c|c|c|c|}
\hline \multicolumn{2}{|l|}{ Findings } & \multirow{2}{*}{$\begin{array}{c}\text { Cases } \\
9\end{array}$} & \multirow{2}{*}{$\frac{\text { Percent }}{24.3 \%}$} \\
\hline Colloid & None & & \\
\hline & Scanty & 15 & $40.5 \%$ \\
\hline & Moderate & 5 & $13.5 \%$ \\
\hline & Abundant & 8 & $21.6 \%$ \\
\hline \multirow[t]{4}{*}{ Cellularity } & None & 0 & $0 \%$ \\
\hline & Scanty & 9 & $24.3 \%$ \\
\hline & Moderate & 10 & $27.0 \%$ \\
\hline & Abundant & 18 & $48.6 \%$ \\
\hline \multirow[t]{4}{*}{ Arrangement } & Papillary & 0 & $0 \%$ \\
\hline & Microfollicular & 31 & $83.8 \%$ \\
\hline & Normofollicular & 9 & $24.3 \%$ \\
\hline & Macrofollicular & 6 & $16.2 \%$ \\
\hline \multicolumn{2}{|c|}{ Multinucleated giant cells } & 1 & $2.7 \%$ \\
\hline \multicolumn{2}{|l|}{ Lymphocytes } & 1 & $2.7 \%$ \\
\hline \multicolumn{2}{|l|}{ Foamy cells } & 2 & $5.4 \%$ \\
\hline \multicolumn{2}{|c|}{ Metaplastic cytoplasm } & 1 & $2.7 \%$ \\
\hline \multicolumn{2}{|c|}{ Intranuclear cytoplasmic inclusions } & 25 & $67.6 \%$ \\
\hline \multicolumn{2}{|c|}{ Nuclear grooves } & 35 & $94.6 \%$ \\
\hline \multicolumn{2}{|c|}{ Irregular-shaped nuclei } & 35 & $94.6 \%$ \\
\hline \multicolumn{2}{|c|}{ Clear chromatin } & 22 & $59.5 \%$ \\
\hline \multicolumn{2}{|c|}{ Nuclear overlapping } & 7 & $18.9 \%$ \\
\hline
\end{tabular}


cases of NIFTP, respectively (Fig. 3). In cases showing normofollicular or macrofollicular patterns, the $\mathrm{N} / \mathrm{C}$ ratio of the carcinoma cells was low (Fig. 3a), and some nuclei were spindle-shaped. Papillary arrange- ment was not observed. The incidences of intranuclear cytoplasmic inclusions, nuclear grooves (Fig. 4a), irregular-shaped nuclei (Fig. 4b, c), clear chromatin pattern, and nuclear overlapping as nuclear features
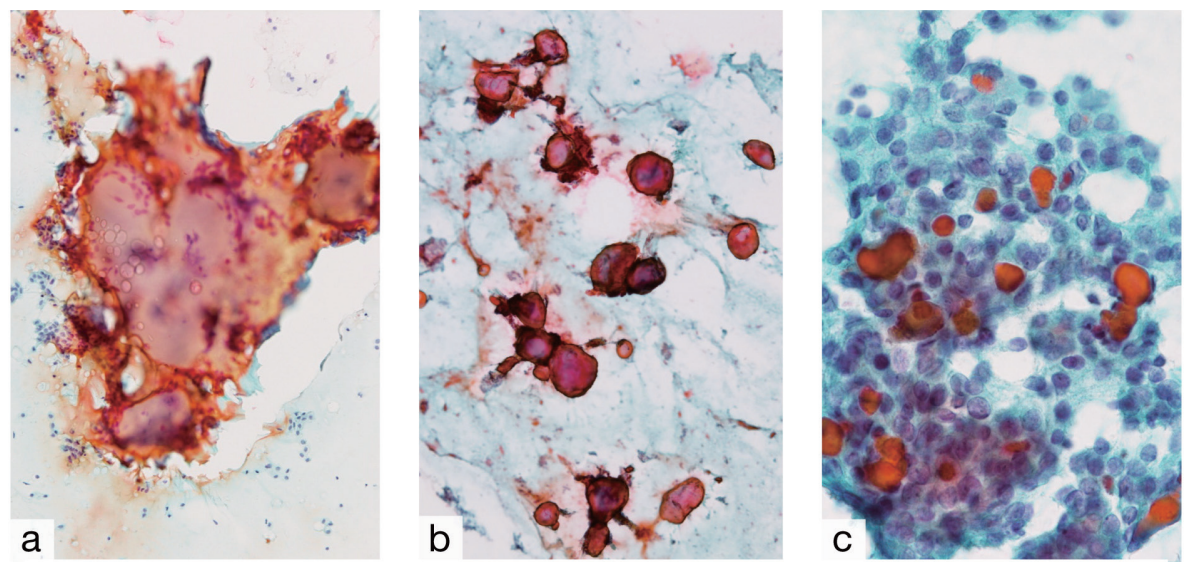

Fig. 2 Colloids of NIFTP exhibited lumps of various sizes (a: large; b: medium; and c: small). (Papanicolaou stain; a: 40×; b: 40×; c: 200×).
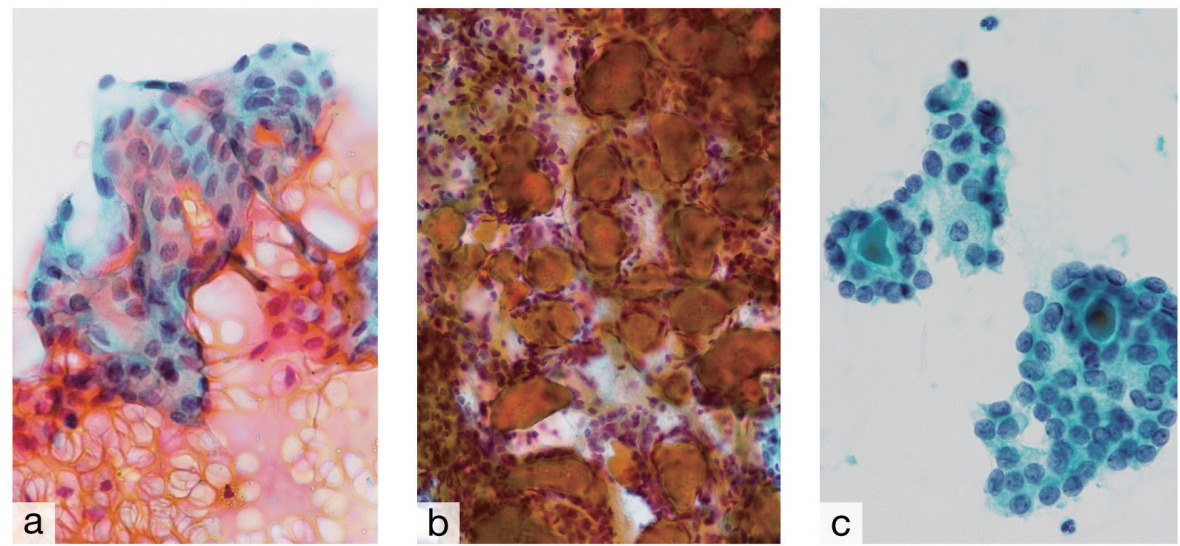

Fig. 3 NIFTP showed (a) macrofollicular, (b) normofollicular, and (c) microfollicular patterns. The nuclear-cytoplasmic ratio of the carcinoma cells showing a macrofollicular pattern was low. (Papanicolaou stain; a: 200×; b: 100×; c: 200×).
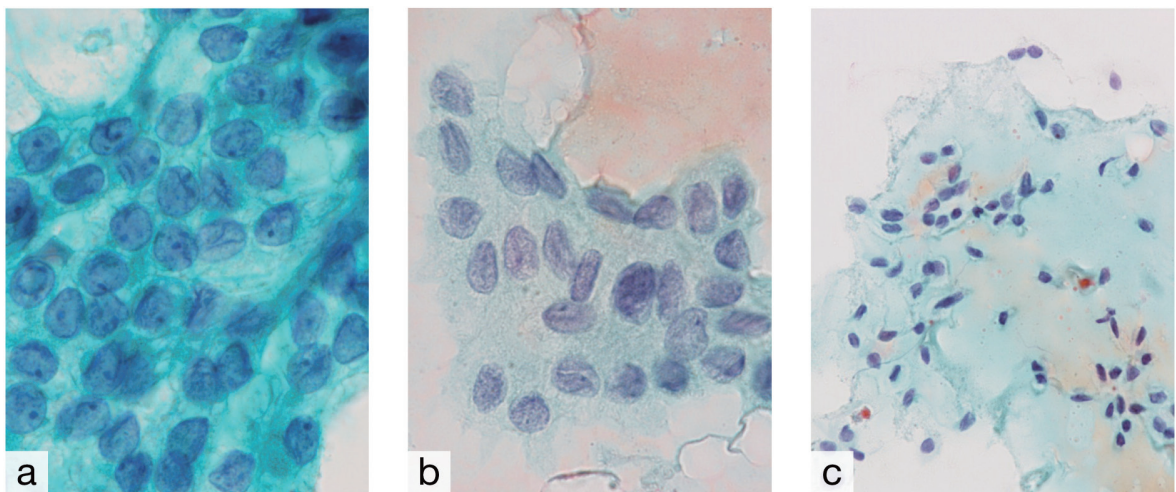

Fig. 4 Nuclei of NIFTP showed (a) grooves, (b) irregular shapes, and (c) spindle shapes. (Papanicolaou stain, a: 1,000×; b: 1,000×; c: 200×). 
indicating PTC were 67.6\%, 94.6\%, 94.6\%, 59.5\%, and $18.9 \%$, respectively.

\section{Discussion}

In 2016, Nikiforov et al. proposed renaming noninvasive encapsulated FV-PTC to NIFTP as a type of non-malignant tumor to prevent overtreatment [1]. Thompson examined 94 cases of encapsulated FV-PTC and did not demonstrate histological features of other known variants, such as tall cell, columnar, oncocytic, insular, or cribriform, of PTC [2]. However, in our study, one-fourth of the total cases of NIFTP were originally diagnosed as noninvasive, encapsulated, macrofollicular variants of PTC. The variants had been also included in $11.2 \%$ of the cases of invasive encapsulated FV-PTC. Such findings had not been reported previously. When the entity of NIFTP is considered, macrofollicular variants of PTC do not include noninvasive cases, and include only invasive cases, such as follicular variant of PTC.

According to a report by Nikiforov et al., the incidences of follicular variants of PTC and NIFTP range from $22.4 \%$ to $43.4 \%$ and from $13.6 \%$ to $25 \%$ of the cases of PTC, respectively [1]. Hahn et al. reported that $34(16.3 \%)$ of 208 cases of FV-PTC were NIFTP [6]. In contrast, in the Asian countries, cases of NIFTP are estimated to represent only $0.8 \%$ cases of PTC [3]. In our study, cases of FV-PTC and NIFTP comprised 2.8\% and $0.5 \%$ cases of PTC, respectively. The incidences of FV-PTC and NIFTP in the Asian countries were considerably lower than those in the western countries. Jung and Kim reported that the incidence of NIFTP diagnosed between 2008 and 2011 was $0.3 \%$ of all the cases of PTC [4]. Subsequently, from 2012, they adopted "loose diagnostic criteria" to define NIFTP, which was then diagnosed at a much higher rate of $3.4 \%$. This large difference in incidences may allow for evaluation of the nuclear features of PTC. However, there is still a major difference between the Asian and western countries. Other factors, such as race, dietary habit, or indication for resection, may be related.

In our study, there were no significant differences in the age, sex, and tumor size between cases of NIFTP and those of invasive encapsulated FV-PTC. However, the incidence of NIFTP tended to be higher than that of invasive FV-PTC in men. This tendency was also reported by Hahn et al., in which the incidences of NIFTP and FV-PTC in men were $35.3 \%$ and $24.1 \%$, respectively [6]. NIFTP was seen on an US investigation typically as a circumscribed oval/round nodule with a hypoechoic rim, while the Doppler investigation showed hypervascularity [8]. Thus, the findings lacked malignant features. The US investigation showed a significantly lower rate $(14.7 \%)$ of high suspicion NIFTP than invasive FV-PTC (37.9\%) [6]. In our study, the rate (6.5\%) of high suspicion NIFTP was significantly lower than that of invasive encapsulated FV-PTC, and half of the cases of NIFTP were interpreted as low suspicion.

The previous reports described that most NIFTP cases were cytologically classified as benign, AUS/ FLUS, or FN/SFN [5, 9-15]. However, in our study, three-fourth of the NIFTP cases were reported as SFM or malignant. Similarly, Hahn et al. reported that the most common cytological diagnosis of NIFTP was SFM, and there were no differences in the diagnosis of SFM and malignancy between NIFTP and invasive encapsulated FV-PTC [6]. We believe that our results were reasonable. The tumor cells aspirated from NIFTP with the nuclear features of PTC should have revealed these features. Thus, it is not logical that the nuclear features of PTC could not be identified on cytological specimens in most cases of NIFTP.

No particular cytological features can reliably distinguish NIFTP from invasive FV-PTC [16-18]. According to previous reports, NIFTP shows a predominantly microfollicular pattern similar to that shown by invasive FV-PTC. Papillary arrangement, psammoma bodies, or abundant colloids were not found. The incidences of intranuclear cytoplasmic inclusions, nuclear grooves, overlapping nuclei, nuclear elongation, and multinucleated giant cells are lower than those of conventional PTC [18]. However, Maletta et al. reported that nuclear features of NIFTP were not significantly different from those of invasive FV-PTC [17]. In our study, nuclear grooves and irregularly shaped nuclei were observed in $94.6 \%$ of cases of NIFTP. These findings led us to the diagnosis of PTC and were significant even in cases of NIFTP (the noninvasive macrofollicular variant) that were apparently benign because of the low nuclear/cytoplasmic ratio, macrofollicular pattern, and abundant colloids. We believe that nuclear grooves and irregular nuclei might be the most useful findings leading to the cytological diagnosis of NIFTP. Such findings may be overlooked in cases of NIFTP that had been classified into intermediate categories.

It is impossible to distinguish NIFTP from invasive FV-PTC based on FNAC. Therefore, NIFTP cannot be 
pre-operatively diagnosed. The diagnosis of NIFTP can only be carried out by histological examination of surgically resected specimens. If the term NIFTP is truly adopted, it will be necessary to provide a new category, i.e., a term "tumor with papillary-like nuclear features", in BSRTC that includes NIFTP and invasive FV-PTC, similar to FN/SFN, including follicular adenoma and follicular carcinoma [7]. For the clinical management, lobectomy should be recommended, similar to FN/SFN. Total thyroidectomy, lymph node dissection, or radioactive iodine ablation treatment are not necessary. Therefore, we concluded that NIFTP should be renounced noninva- sive encapsulated FV-PTC, and should be considered as a malignant tumor with exceeding indolent behavior, and lobectomy alone should be satisfactory for the diagnosis and treatment. Active surveillance may be considered as a strategy for clinical management, similar to low-risk papillary microcarcinoma [19].

\section{Disclosure}

The authors have no conflicts of interest to declare regarding grant support or financial relationships.

\section{References}

1. Nikiforov YE, Seethala RR, Tallini G, Baloch ZW, Basolo F, et al. (2016) Nomenclature revision for encapsulated follicular variant of papillary thyroid carcinoma: a paradigm shift to reduce overtreatment of indolent tumors. JAMA Oncol 2: 1023-1029.

2. Thompson LD (2016) Ninety-four cases of encapsulated follicular variant of papillary thyroid carcinoma: a name change to noninvasive follicular thyroid neoplasm with papillary-like nuclear features would help prevent overtreatment. Mod Pathol 29: 698-707.

3. Bychkov A, Hirokawa M, Jung CK, Liu Z, Zhu Y, et al. (2017) Low rate of NIFTP in Asian practice. Thyroid 27: 983-984.

4. Jung CK, Kim C (2017) Effect of lowering the diagnostic threshold for encapsulated follicular variant of papillary thyroid carcinoma on the prevalence of noninvasive follicular thyroid neoplasm with papillarylike nuclear features: a single-institution experience in Korea. J Basic Clin Med 6: 26-28.

5. Brandler TC, Zhou F, Liu CZ, Cho M, Lau RP, et al. (2017) Can noninvasive follicular thyroid neoplasm with papillary-like nuclear features be distinguished from classic papillary thyroid carcinoma and follicular adenomas by fine-needle aspiration? Cancer 125: 378-388.

6. Hahn SY, Shin JH, Lim HK, Jung SL, Oh YL, et al. (2017) Preoperative differentiation between noninvasive follicular thyroid neoplasm with papillarylike nuclear features (NIFTP) and non-NIFTP. Clin Endocrinol (Oxf) 86: 444-450.

7. Cibas ES, Ali SZ (2009) The Bethesda System for Reporting Thyroid Cytopathology. Thyroid 19: 1159-1165.

8. Yang GC, Fried KO, Scognamiglio T (2017) Sonographic and cytologic differences of NIFTP from infiltrative or invasive encapsulated follicular variant of papillary thyroid carcinoma: a review of 179 cases.
Diagn Cytopathol 45: 533-541.

9. Canberk S, Baloch ZW, Ince U, Schmitt F (2017) Diagnosis of non-invasive follicular tumor with papillary-like nuclear features (NIFTP): a practice changer for thyroid fine-needle aspiration interpretation. J Basic Clin Med 6: 38-43.

10. Faquin WC, Wong LQ, Afrogheh AH, Ali SZ, Bishop JA, et al. (2016) Impact of reclassifying noninvasive follicular variant of papillary thyroid carcinoma on the risk of malignancy in The Bethesda System for Reporting Thyroid Cytopathology. Cancer Cytopathol 124: 181-187.

11. Canberk S, Gunes P, Onenerk M, Erkan M, Kilinc E, et al. (2016) New concept of the encapsulated follicular variant of papillary thyroid carcinoma and its impact on the Bethesda System for Reporting Thyroid Cytopathology: a single-institute experience. Acta Cytol 60: 198-204.

12. Baloch ZW, Seethala RR, Faquin WC, Papotti MG, Basolo F, et al. (2016) Noninvasive follicular thyroid neoplasm with papillary-like nuclear features (NIFTP): A changing paradigm in thyroid surgical pathology and implications for thyroid cytopathology. Cancer Cytopathol 124: 616-620.

13. Wu HH (2017) The impact of NIFTP on FNA cytology: can we still diagnose papillary thyroid carcinoma? J Basic Clin Med 6: 61-62.

14. Jeon MJ, Song DE, Jung CK, Kim WG, Kwon H, et al. (2016) Impact of reclassification on thyroid nodules with architectural atypia: from non-invasive encapsulated follicular variant papillary thyroid carcinomas to non-invasive follicular thyroid neoplasm with papillary-like nuclear features. PLoS One 11: e0167756.

15. Ibrahim AA, Wu HH (2016) Fine-needle aspiration cytology of noninvasive follicular variant of papillary thyroid carcinoma is cytomorphologically distinct from the inva- 
sive counterpart. Am J Clin Pathol 146: 373-377.

16. Strickland KC, Vivero M, Jo VY, Lowe AC, Hollowell $\mathrm{M}$, et al. (2016) Preoperative cytologic diagnosis of noninvasive follicular thyroid neoplasm with papillaryLike nuclear features: a prospective analysis. Thyroid 26: 1466-1471.

17. Maletta F, Massa F, Torregrossa L, Duregon E, Casadei GP, et al. (2016) Cytological features of "noninvasive follicular thyroid neoplasm with papillary-like nuclear features" and their correlation with tumor histology.
Hum Pathol 54: 134-142.

18. Ng D, Can NT, Ma ZV, Zante AV, Ljung BM, et al. (2017) Cytomorphologic features of noninvasive follicular thyroid neoplasm with papillary-like nuclear features (NIFTP): a comparison with infiltrative follicular variant of papillary thyroid carcinoma. J Basic Clin Med 6: 51-56.

19. Miyauchi A (2016) Clinical trials of active surveillance of papillary microcarcinoma of the thyroid. World $J$ Surg 40: 516-522. 